\title{
Ecological Status of Opa Reservoir, Obafemi Awolowo University, Ile Ife, based on the Abundance and Diversity of its Planktonic Flora
}

\author{
Oluseun Peter BOLAWA ${ }^{1}$, Adebukola Adenike ADEDEJI ${ }^{1 *}$, \\ Yetunde Folasade TAIWO ${ }^{2}$ \\ ${ }^{1}$ Obafemi Awolowo University, Department of Zoology, Ile-Ife, \\ Nigeria; bbkadedeji@yahoo.com; bbkadedeji@oauife.edu.ng(*correspondingauthor) \\ ${ }^{2}$ Natural History Museum, Obafemi Awolowo University, Ile-Ife, Nigeria
}

\begin{abstract}
A study investigating the spatial and temporal distribution, composition and abundance of plankton in Opa reservoir, Obafemi Awolowo University, Ile-Ife, Southwest Nigeria, was conducted over a period of an annual cycle. The study was undertaken with a view of providing a more recent catalogue of planktonic flora and possibly an update of the reservoir's trophic status. Quantitative net planktons were collected monthly from both the surface and bottom levels at three sampling stations established at the dam site (lacustrine), mid-lake (transition) and upper inflow (riverine) parts of the reservoir. The divisions recorded were Bacillariophyta $>$ Cyanophyta $>$ Chlorophyta $>$ Euglenophyta $>$ Myzozoa $>$ Ochrophyta $=$ Charophyta $>$ Cryptophyta in order of abundance. Vertically, the highest occurrence of species was recorded at the lacustrine bottom station (71 species), while the least occurrence was observed in the transition bottom station ( 51 species). A total of sixteen plankton species showed significant seasonal variation in abundance during this study period, while only seven species had significant spatial variation $(\mathrm{p} \leq 0.05)$. Higher abundance was observed during the rainy season $\left(170,797,350 \mathrm{Org} / \mathrm{m}^{3}\right.$ from seventy-two species) than dry season $\left(5,138,400 \mathrm{Org} / \mathrm{m}^{3}\right.$ from forty-nine species). Notable bio-indicator plankton species recorded were Anabaena circinalis, Anabaena flos-aquae, Microcystis sp., Aphanocapsa litoralis and Microcystis aeruginosa. Some other pollution indicator species recorded were Synedra ulna, Oscillatoria agardhii, Phacus sp., Surirella sp., Closterium sp., Aphanocapsa sp. and Euglena sp. Hence, Opa reservoir is very rich in Bacillariophyta (diatoms), followed by Cyanophyta (bluegreen) and Chlorophyta (green algae), which are known to characterize eutrophic lakes.
\end{abstract}

Keywords: bio-indicator species; phytoplankton; taxonomic composition; trophic status; water quality

\section{Introduction}

By the virtue of the position of phytoplankton at the base of the aquatic food web, they stand as the most important factor of production in the aquatic ecosystem (Moshood, 2009). Various ecological changes such as presence, absence, replacement or addition of species can also be monitored using the phytoplanktonic community as a potential tool (Codd, 1995). Therefore, the presence of phytoplankton in reservoirs goes a long way in determining the sustainability and productivity of most aquatic habitats. The growth significance and sustainability of any ecosystem is largely accounted for by the diversities of phytoplankton and their abundance. Both factors are equally related and do change as their interaction is influenced by the environment and population processes (Benedict and Gabriel, 2012).

Phytoplankton are known to be very important in estimation of the potential fish yield (Hecky and Kling, 1981), productivity (Park et al., 2003), water quality (Walsh et al., 2001), energy flow (Simciv, 2005), trophic status (Reynolds, 1999), and water management (Beyruth, 2000). Phytoplankton such as Microcytis sp., Anabaena sp., Oscillatoria sp. are known indicators of pollution while the presence and abundance of Chlorophyceae are indicative of the environment's suitability for fish production (Olasehinde and Abeke, 2012). The suitability of microalgal components as bio-indicators of the water condition is because they confer more tolerance than many other biotas used for monitoring environmental changes (Nwankwo and Akinsoji, 1992). 
288

Adesakin et al. (2017) reported direct discharge of untreated municipal/industrial waste as well as run off from agricultural areas into Opa reservoir, with resultant significant effects on the reservoir's physicochemical parameters both temporally and spatially and this may possibly inflict a level of risk to the inhabiting aquatic biota. This, coupled with the fact that the last published record of plankton research carried out on Opa reservoir was that of Rotifers only by Akinbuwa and Adeniyi (1996), lead to the present study. The study seeks to determine the taxonomic composition, diversity and abundance of phytoplanktonic organisms of Opa reservoir with respect to spatial and temporal distribution, as well as to assess the water quality and trophic status of the reservoir with a view to determining the effects of the discharges.

\section{Materials and Methods}

\section{Plantmaterial}

The study site, Opa reservoir (Fig. 1), is located between longitude $004^{\circ} 31^{\prime} 40^{\prime \prime} \mathrm{E}$ to $004^{\circ} 32^{\prime} 45^{\prime \prime} \mathrm{E}$, and latitude $07^{\circ} 30^{\prime} \mathrm{N}$ to $07^{\circ} 31^{\prime} \mathrm{N}$, within the Obafemi Awolowo University community, Ile-Ife, Southwest Nigeria (Fawole and Arawomo, 2000). The reservoir was established in 1978 by the impoundment of River Opa which sources from OkeOpa, a set of hills on the Eastern side of the Ife/Ilesha road, Ile-Ife, Osun state (Akinbuwa and Adeniyi, 1996). A number of rivers, including Amuta, Esinmirin, Obudu and Opa unite to form the Opa River. The reservoir has a catchment basin of about $116 \mathrm{~km}^{2}$ (Akinbuwa and Adeniyi, 1996). Its total surface area is $0.95 \mathrm{~km}^{2}$, while the maximum capacity is about $675,000 \mathrm{~m}^{3}$ with depth of $0.95 \mathrm{~m}$ and 6.4 $\mathrm{m}$ at littoral zone and open water respectively (Fawole and Arawomo, 2000). The dam wall made of gravel is about $0.28 \mathrm{~km}$ long and about $15 \mathrm{~m}$ from the foundation to the crest (Akinbuwa and Adeniyi, 1991). As expected of tropical shallow reservoirs, the water volume during the dry season reduces significantly, whereas in the rainy season, there is increased volume of water inflow resulting from floods leading to high turbidity and a general immersion of the vegetation on the shoreline. This seasonal fluctuation in the water discharge into the reservoir directly affects its water level.

Three sampling stations A, B and C were established on the reservoir denoting the lacustrine, transition and the riverine area of the reservoir along the horizontal axis respectively (Fig. 1). The station $\mathrm{A}$ is located at the dam-site just beside the wall, an area assumed to be the deepest part of the lake, station B is the middle of the lake, while the $\mathrm{C}$ station is towards the inflow into the lake. A permanent buoy (rubber float) was used to demarcate each of the three sampling stations for ease of subsequent recognition. The distances between the stations and the grid coordinate of each station was taken and recorded using the Global Positioning System (GPS) handheld receiver.

\section{Sample collection}

Water samples were collected monthly from both the surface and bottom levels at the three sampling stations on the reservoir for a period of one year for phytoplankton analysis between October 2012 and November 2013. An improvised water sampler of $2.5 \mathrm{~L}$ capacity was used to take bottom water samples at required depths. Net plankton was sampled by pouring 20 litres of water through plankton net of $50 \mu \mathrm{m}$ mesh size and the net planktonic contents was poured into a $30 \mathrm{ml}$ sampling bottle and preserved with few drops of 5\% formaldehyde and a drop of Lugol's solution for examination and identification. The preserved subsample containing plankton was examined in the laboratory using OMAX binocular light compound photo and their scaled pictures taken.

Planktonic population abundance was estimated based on the count records of the final concentrate volume of the

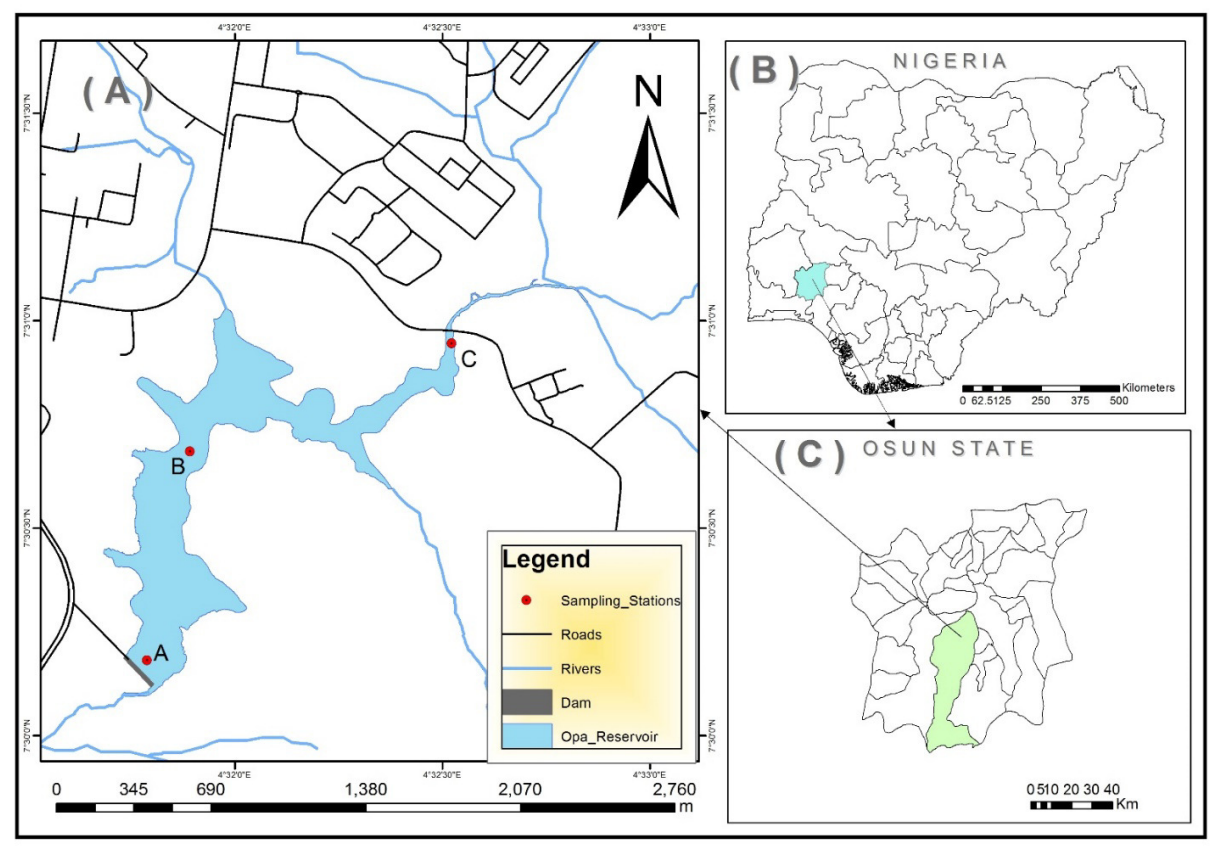

Fig. 1. Map of Opa Reservoir showing the investigated sampling stations (A); Nigeria (B); Osun State (C) 
sub-sample with respect to the original volume of water filtered with plankton net and the result was then expressed in organisms per cubic metre of the original water sample.

\section{Data analysis}

Data collected were subjected to various descriptive and inferential analyses such as the means and standard deviations which gave the depiction of planktonic species abundance with respect to season and location. Analysis of variance was used to compare mean abundance of identified planktonic species, while correlation was used to show the relationship between different planktonic groups. Moreover, Principal Component Analysis (PCA) was used to reduce all interactions into components that also showed the relationship between recorded plankton species as applicable using SPSS Version 21 software (SPSS, 2012). Plankton community structure was determined using Species diversity indices (Shannon and Weaver, 1949), Dominance (Magurran, 2004), Species equitability or evenness (Pielou, 1966) and Species richness (Margalef, 1951; Menhinick, 1964).

\section{Results}

\section{Species composition and occurrence}

A total of eighty-two (82) species of phytoplankton were recorded belonging to fifty-five genera, forty-five families, thirty-two orders, twelve classes and eight divisions of algae as outlined below in Table 1 and summarized in Fig. 2. A total of 38 species occurred at all the three sampling stations at the surface and/or the bottom. These species include seven species of Cyanobacteria division (Anabaena circinalis, Cylindrospermopsis raciborskii, Microcystis sp., Microcytis aeruginosa, Trachodesmium lacustre, Oscillatoria agardhii and Coelosphaerium sp.). Others are seven species belonging to Chlorophyta (Oedogonium sp., Pediastrum simplex, Pediastrum sp., P. duplex, Volvox aureus, Actinastrum hantzschii and Oocystis sp.) and three species (Euglena oxyuris, Euglena acus and Phacus longicauda) representing Euglenophyta. Furthermore, species found only once throughout the sampling period and specific to a sampling station include Anabaena flos-aquae, Chroococcus sp., Lyngbya sp., Cosmarium depressum, Cosmarium subcrenatum, Schizothrix lardacea, Aphanocapsa litoralis, (Lacustrine); Melosira oamaruensis, Guarnardia flaccida, Thalasionema sp., Prasiola sp., Peridinopsis thompsonii, Trachelomonas caudata (Transition) and Melosira sp. (Riverine).

\section{Spatial variation}

Phytoplankton total abundance ranged from $15,855,150 \mathrm{Org} / \mathrm{m}^{3}$ at transition (surface) to $53,956,350$ $\mathrm{Org} / \mathrm{m}^{3}$ at the lacustrine zone (surafce) of the reservoir. The recorded abundance as compared with zooplankton abundance recorded during the study period showed an average of 112 times (5.71-175.04 times) higher phytoplankton than the zooplankton recorded (Table 2).

The maximum mean abundance was also recorded at the lacustrine surface portion of the reservoir $(963,506$ $\mathrm{Org} / \mathrm{m}^{3}$ ) which had highest number of species (56 species) (Table 2). Horizontally, Pediastrum sp., Actinastrum hantzschii and Dinophysis sp. showed significant difference across zones ( $\mathrm{p} \leq 0.05$ ), while Amphipleura jenneri, Pediastrum simplex, Pediastrum duplex and Volvox aureus showed highly significant spatial difference $(\mathrm{p} \leq 0.01)$ (Table 3).

\section{Seasonal variation}

Higher abundance was observed during the rainy season $\left(170,797,350 \mathrm{Org} / \mathrm{m}^{3}\right.$ from seventy-two species) than dry season $\left(5,138,400 \mathrm{Org} / \mathrm{m}^{3}\right.$ from forty-nine species) (Table 2 ). The most abundant species during the dry season include Guinardia flaccida, Cylindrospermopsis raciborskii, Oscillatoria agardhii, Perdiniopsis pernardii, Pediastrum simplex and Oedogonium sp. (Table 3). Species showing highly significant seasonal difference $(\mathrm{p} \leq 0.01)$ include Nitzschia sp., Pediastrum simplex, Pediastrum sp., Pediastrum duplex, Volvox aureus and Dinophysis sp. While Amphipleura jenneri, Surirella tenara, Chaetocerous subtilis, Anabaena circinalis, Microcystis aeruginosa, Ceratium inflatum, Euglena oxyuris and Trachelomonas caudata all showed statistically signicant difference seasonally $(\mathrm{p} \leq$ 0.05) (Table 4).

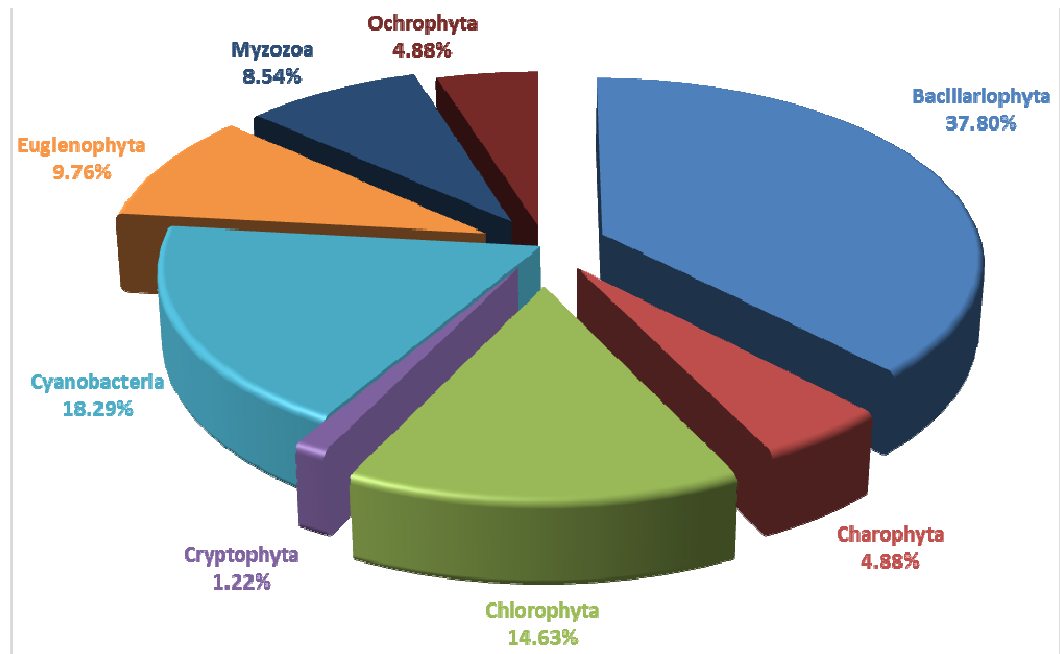

Fig. 2. Percentage composition of recorded phytoplankton taxa 
Table 1. Outline classification and taxa composition of the phytoplankton flora

\begin{tabular}{|c|c|c|c|c|c|}
\hline Division & Class & Subclass & Order & Family & Genus/Species \\
\hline \multirow{15}{*}{ Cyanobacteria } & Cyanophyceae & Nostocophycidae & Nostocales & Nostocaceae & Anabaena circinalis \\
\hline & & & & & Anabaena flos-aquae \\
\hline & & & & Aphanizomenonaceae & Cylindrospermopsis raciborskii \\
\hline & & Oscillatoriophycidae & Chroococcales & Chroococcaceae & Chroococcus sp. \\
\hline & & & & Microcystaceae & Microcystis sp. \\
\hline & & & & & Microcystis aerosonosa \\
\hline & & & Oscillatoriales & Gloeotrichiaceae & Gloeotrichia echinulata \\
\hline & & & & Microcoleaceae & Arthrospira sp. \\
\hline & & & & & Trachodesmium lacustre \\
\hline & & & & Oscillatoriaceae & Oscillatoria agardhii \\
\hline & & & & & Lyngbya contorta \\
\hline & & & & & Lyngbya sp. \\
\hline & & Synechococcophycideae & Pseudanabaenales & Schizotrichaceae & Schizothrix lardacea \\
\hline & & & Synechococcales & Merismopediaceae & Aphanocapsa litoralis \\
\hline & & & & Coelosphaeriaceae & Coelosphaerium sp. \\
\hline \multirow[t]{12}{*}{ Chlorophyta } & Chlorophyceae & & Oedogoniales & Oedogoniaceae & Oedogonium sp. \\
\hline & & & Sphaeropleales & Hydrodictyaceae & Pediastrum duplex \\
\hline & & & & & Pediastrum simplex \\
\hline & & & & & Pediastrum sp. \\
\hline & & & & & Hydrodictyon reticulatum \\
\hline & & & Chlamydomonadales & Volvocaceae & Volvox globulus \\
\hline & & & & & Volvox aureus \\
\hline & Trebouxiophyceae & & Chlorelalles & Chlorellaceae & Actinastrum hantzschii \\
\hline & & & & & Dictyosphaerium sp. \\
\hline & & & & Oocystaceae & Oocystis crassa \\
\hline & & & & & Oocystis sp. \\
\hline & & & Prasiolales & Prasiolaceae & Prasiola sp. \\
\hline \multirow[t]{8}{*}{ Euglenophyta } & Euglenophyceae & & Euglenales & Euglenaceae & Euglena sp. \\
\hline & & & & & Euglena oxyuris \\
\hline & & & & & Euglena viridis \\
\hline & & & & & Euglena acus \\
\hline & & & & & Trachelomonas sp. \\
\hline & & & & & Trachelomonas caudata \\
\hline & & & & Phacaceae & Phacus longicauda \\
\hline & 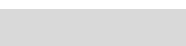 & & & & Phacus pyrum \\
\hline \multirow[t]{7}{*}{ Myzozoa } & Dinophyceae & & Perdianiales & Perdiniaceae & Peridinium sp. \\
\hline & & & & & Peridinium willei \\
\hline & & & & & Peridinium cinctum \\
\hline & & & & Glenodiniaceae & Peridiniopsis pernardii \\
\hline & & & & & Peridiniopsis thompsonii \\
\hline & & & Gonyaulacales & Ceratiaceae & Ceratium inflatum \\
\hline & & & Dinophysiales & Dinophysaceae & Dinophysis sp. \\
\hline Cryptophyta & Cryptophyceae & & Cryptomonadales & Cryptomonadaceae & Cryptomonas ovata \\
\hline \multirow[t]{4}{*}{ Ochrophyta } & Synurophyceae & & Synurales & Mallomonadaceae & Mallomonas caudata \\
\hline & & & Desmidiales & Desmidiaceae & Desmidium sp. \\
\hline & & & & & Cosmarium depressum \\
\hline & & & & & Cosmarium subcrenatum \\
\hline \multirow[t]{4}{*}{ Charophyta } & Conjugatophyceae & & Zygnematales & Closteriaceae & Closterium sp. \\
\hline & & & & & Closterium lanceolatum \\
\hline & & & & Zygnemataceae & Spirogyra setiformis \\
\hline & & & & & Mougeotia boodlei \\
\hline \multirow[t]{8}{*}{ Bacillariophyta } & Bacillariophyceae & Bacillariophycidae & Naviculales & Amphipleuraceae & Amphipleura jenneri \\
\hline & & & & & Frustulia sp. \\
\hline & & & & Naviculaceae & Navicula sp. 1 \\
\hline & & & & & Navicula sp. 2 \\
\hline & & & & & Navicula sp. 3 \\
\hline & & & & Pleurosigmataceae & Pleurosigma sp. \\
\hline & & & & Stauroneidaceae & Stauroneis sp. \\
\hline & & & & Pinnulariaceae & Pinnularia viridis \\
\hline & & & & & Pinnularia sp. \\
\hline
\end{tabular}




\begin{tabular}{|c|c|c|c|c|}
\hline & & Bacillariales & Bacillariaceae & Nitzschia sigma \\
\hline & & & & Nitzschia sp. \\
\hline & & & & Bacillaria sp. \\
\hline & & & & Bacillaria paradoxa \\
\hline & & Cocconeidales & Cocconeidaceae & Cocconeis pediculus \\
\hline & & Surirellales & Surirellaceae & Surirella tenara \\
\hline & & & & Surirella minuta \\
\hline & Eunotiophycidae & Eunotiales & Eunotiaceae & Eunotia formica \\
\hline Coscinodiscophyceae & Melosirophycidae & Melosirales & Melosiraceae & Melosira oamaruensis \\
\hline & & & & Melosira sp. \\
\hline & & & Coscinodiscaceae & Hyalodiscus sp. \\
\hline & & & & Hyalodiscus radiatus \\
\hline & & Rhizosoleniales & Rhizosoleniaceae & Rhizosolenia sp. \\
\hline & & & & Guinardia flaccida \\
\hline Fragilariophyceae & & Fragilariales & Fragilariaceae & Fragilaria capucina \\
\hline & & Licmophorales & Ulnariaceae & Synedra ulna \\
\hline & & Thalassionematales & Thalassionemataceae & Thalassionema sp. \\
\hline & & & & Thalassionema nitzschioides \\
\hline Mediophyceae & Chaetocerotophycidae & Chaetocerotales & Chaetocerotaceae & Chaetocerous subtilis \\
\hline & Thalassiosirophycidae & Leptocylindrales & Leptocylindraceae & Leptocylindrus danicus \\
\hline & & & & Leptocylindrus minimus \\
\hline & & Thalassiosirales & Stephanodiscaceae & Stephanodiscus sp. \\
\hline
\end{tabular}

Table 2. Spatial and temporal abundance distribution of phytoplankton species

\begin{tabular}{|c|c|c|c|c|c|c|c|c|}
\hline \multirow{3}{*}{ Organisms } & \multirow{2}{*}{\multicolumn{2}{|c|}{ Temporal }} & \multicolumn{6}{|c|}{ Spatial } \\
\hline & & & \multicolumn{2}{|c|}{ Lacustrine } & \multirow{2}{*}{$\begin{array}{c}\text { Transition } \\
\text { Surface }\end{array}$} & \multicolumn{3}{|c|}{ Riverine } \\
\hline & $\begin{array}{c}\text { Dry } \\
\text { season }\end{array}$ & Wet season & Surface & Bottom & & Bottom & Surface & Bottom \\
\hline \multicolumn{9}{|c|}{ Cyanobacteria } \\
\hline Anabaena circinalis & 730,050 & 38,550 & 173,700 & 30,000 & 113,850 & 405,750 & 24,450 & 20,850 \\
\hline Anabaena flos-aquae & 0 & 150 & 0 & 150 & 0 & 0 & 0 & 0 \\
\hline $\begin{array}{c}\text { Cylindrospermopsis } \\
\text { raciborskii }\end{array}$ & 75,600 & $3,709,650$ & $1,349,100$ & 717,000 & $1,086,900$ & 155,400 & 36,750 & 440,100 \\
\hline Chroococcus sp. & 150 & 0 & 0 & 150 & 0 & 0 & 0 & 0 \\
\hline Microcystis sp. & 44,400 & 41,700 & 24,450 & 3,900 & 24,900 & 5,100 & 26,250 & 1,500 \\
\hline Microcystis aeruginosa & 12,900 & 1,050 & 2,250 & 0 & 7,500 & 150 & 3,150 & 900 \\
\hline Gloeotrichia echinulata & 0 & 600 & 300 & 0 & 0 & 0 & 150 & 150 \\
\hline Arthrospira sp. & 1,050 & 300 & 1,050 & 150 & 0 & 0 & 0 & 150 \\
\hline Trachodesmium lacustra & 1,950 & 1,350 & 1,050 & 150 & 600 & 600 & 600 & 300 \\
\hline Oscillatoria agardhii & $1,052,100$ & $12,399,150$ & $5,510,550$ & 766,500 & 96,600 & $6,589,950$ & 185,700 & 301,950 \\
\hline Lyngbya contorta & 0 & 450 & 0 & 150 & 0 & 0 & 300 & 0 \\
\hline Lyngbya sp. & 0 & 150 & 0 & 150 & 0 & 0 & 0 & 0 \\
\hline Schizothrix lardacea & 0 & 150 & 150 & 0 & 0 & 0 & 0 & 0 \\
\hline Aphanocapsa litoralis & 0 & 150 & 0 & 150 & 0 & 0 & 0 & 0 \\
\hline Coelosphaerium sp. & 3,000 & 3,150 & 3,450 & 150 & 300 & 0 & 2,100 & 150 \\
\hline \multicolumn{9}{|c|}{ Chlorophyta } \\
\hline Oedogonium sp. & 31,200 & 269,100 & 12,300 & 170,850 & 450 & 103,500 & 0 & 13,200 \\
\hline Pediastrum simplex & 303,600 & 188,100 & 171,900 & 96,600 & 106,800 & 28,200 & 75,150 & 13,050 \\
\hline Pediastrum sp. & 110,850 & 52,650 & 56,400 & 30,150 & 30,150 & 27,900 & 14,100 & 4,800 \\
\hline Pediastrum duplex & 52,650 & 22,050 & 47,550 & 4,800 & 13,650 & 450 & 7,500 & 750 \\
\hline Hydrodictyon reticulatum & 0 & 300 & 0 & 150 & 0 & 0 & 0 & 150 \\
\hline Volvox globulus & 0 & 1,800 & 750 & 0 & 0 & 0 & 1,050 & 0 \\
\hline Volvox aureus & 5,100 & 150 & 750 & 150 & 450 & 0 & 2,100 & 1,800 \\
\hline Actinastrum hantzschii & 4,950 & 450 & 300 & 150 & 1,200 & 3,450 & 150 & 150 \\
\hline Dictyosphaerium sp. & 0 & 750 & 450 & 0 & 300 & 0 & 0 & 0 \\
\hline Oocystis crassa & 4,500 & 0 & 4,200 & 0 & 0 & 0 & 300 & 0 \\
\hline Oocystis sp. & 900 & 21,300 & 1,350 & 750 & 7,050 & 0 & 5,850 & 7,200 \\
\hline Prasiola sp. & 0 & 300 & 0 & 300 & 0 & 0 & 0 & 0 \\
\hline \multicolumn{9}{|c|}{ Euglenophyta } \\
\hline Euglena sp. & 600 & 2,850 & 450 & 900 & 900 & 600 & 0 & 600 \\
\hline Euglena oxyuris & 16,350 & 4,350 & 1,800 & 9,150 & 1,050 & 450 & 0 & 8,250 \\
\hline Euglena sp. & 750 & 0 & 0 & 600 & 0 & 0 & 150 & 0 \\
\hline Euglena acus & 36,300 & 92,550 & 25,650 & 26,400 & 30,900 & 2,700 & 28,500 & 14,700 \\
\hline
\end{tabular}




\begin{tabular}{|c|c|c|c|c|c|c|c|c|}
\hline \multicolumn{9}{|l|}{292} \\
\hline Trachelomonas sp. & 1,800 & 0 & 0 & 0 & 150 & 0 & 1,650 & 0 \\
\hline T. caudata & 0 & 150 & 0 & 150 & 0 & 0 & 0 & 0 \\
\hline Phacus longicauda & 4,350 & 33,750 & 11,850 & 6,900 & 15,150 & 300 & 3,600 & 300 \\
\hline Phacus pyrum & 0 & 7,500 & 900 & 0 & 6,300 & 0 & 300 & 0 \\
\hline \multicolumn{9}{|c|}{ Myzozoa } \\
\hline Peridinium sp. & 3,900 & 208,050 & 57,450 & 96,450 & 18,000 & 2,850 & 35,400 & 1,800 \\
\hline Peridinium willei & 0 & 3,450 & 1,950 & 150 & 1,200 & 0 & 0 & 150 \\
\hline Peridinium cinctum & 0 & 32,850 & 18,000 & 7,350 & 6300 & 450 & 600 & 150 \\
\hline Peridiniopsispernardii & 714,150 & $5,266,200$ & $1,034,550$ & $1,608,150$ & $3,337,350$ & 0 & 0 & 300 \\
\hline Peridiniopsis thompsonii & 0 & 150 & 0 & 0 & 0 & 0 & 150 & 0 \\
\hline Ceratium inflatum & 1,350 & 0 & 0 & 0 & 0 & 450 & 300 & 600 \\
\hline Dinophysis sp. & 99,450 & 51,450 & 30,300 & 24,150 & 20,100 & 12,900 & 25,800 & 37,650 \\
\hline \multicolumn{9}{|c|}{ Cryptophyta } \\
\hline Cryptomonas ovata & 22,950 & 100,500 & 17,250 & 12,900 & 8,100 & 9,150 & 69,750 & 6,300 \\
\hline \multicolumn{9}{|l|}{ Ochrophyta } \\
\hline Mallomonas caudata & 0 & 450 & 0 & 300 & 150 & 0 & 0 & 0 \\
\hline Desmidium sp. & 5,100 & 145,200 & 25,200 & 4,800 & 300 & 120,000 & 0 & 0 \\
\hline Cosmarium depressum & 0 & 300 & 0 & 300 & 0 & 0 & 0 & 0 \\
\hline Cosmarium subcrenatum & 0 & 150 & 0 & 150 & 0 & 0 & 0 & 0 \\
\hline \multicolumn{9}{|c|}{ Charophyta } \\
\hline Closterium sp. & 0 & 300 & 150 & 0 & 150 & 0 & 0 & 0 \\
\hline C. lanceolatum & 600 & 450 & 450 & 0 & 150 & 450 & 0 & 0 \\
\hline Spirogyra setformis & 0 & 1,200 & 0 & 750 & 0 & 0 & 150 & 300 \\
\hline Mougeotia boodlei & 0 & $14,554,200$ & 580,650 & $13,431,300$ & 48,450 & 144,300 & 15,750 & 333,750 \\
\hline \multicolumn{9}{|c|}{ Bacillariophyta } \\
\hline Amphipleura jenneri & 750 & 0 & 300 & 450 & 0 & 0 & 0 & 0 \\
\hline Frustulia sp. & 0 & 150 & 150 & 0 & 0 & 0 & 0 & 0 \\
\hline Navicula sp. 1 & 600 & 1,800 & 150 & 1,500 & 150 & 450 & 0 & 150 \\
\hline Navicula sp. 2 & 150 & 150 & 0 & 0 & 0 & 0 & 150 & 150 \\
\hline Navicula sp. 3 & 0 & 150 & 0 & 0 & 0 & 0 & 150 & 0 \\
\hline Pleurosigma sp. & 0 & 3,150 & 0 & 1,650 & 0 & 1,500 & 0 & 0 \\
\hline Stauroneis sp. & 300 & 150 & 0 & 0 & 0 & 0 & 0 & 450 \\
\hline Pinnularia viridis & 150 & 10,200 & 8,700 & 0 & 1,500 & 150 & 0 & 0 \\
\hline Pinnularia sp. & 0 & 150 & 0 & 150 & 0 & 0 & 0 & 0 \\
\hline Nitzschia sigma & 750 & 450 & 150 & 150 & 450 & 150 & 0 & 300 \\
\hline Nitzschia sp. & 2,100 & 600 & 450 & 600 & 0 & 300 & 0 & 1,350 \\
\hline Bacillaria sp. & 0 & 300 & 300 & 0 & 0 & 0 & 0 & 0 \\
\hline Bacillaria paradoxa & 0 & 5,400 & 1,350 & 3,450 & 300 & 0 & 0 & 300 \\
\hline Cocconeis pediculus & 300 & 300 & 150 & 0 & 300 & 0 & 0 & 150 \\
\hline Surirella tenara & 3,150 & 0 & 150 & 0 & 0 & 300 & 600 & 2,100 \\
\hline Surirella minuta & 300 & 2,400 & 300 & 2400 & 0 & 0 & 0 & 0 \\
\hline Eunotia formica & 2,250 & 412,350 & 34,950 & 55,500 & 10,650 & 206,250 & 2,250 & 105,000 \\
\hline Melosira oamaruensis & 300 & 300 & 0 & 0 & 300 & 0 & 0 & 300 \\
\hline Melosira sp. & 0 & 150 & 0 & 0 & 0 & 0 & 150 & 0 \\
\hline Hyalodiscus sp. & 0 & 450 & 0 & 0 & 0 & 0 & 450 & 0 \\
\hline Hyalodiscus radiatus & 300 & 450 & 300 & 0 & 0 & 0 & 150 & 300 \\
\hline Rhizosolenia sp. & 0 & 1,200 & 150 & 750 & 0 & 0 & 300 & 0 \\
\hline Guinardia flaccida & $1,750,650$ & $132,891,000$ & $44,681,700$ & $17,239,800$ & $10,782,150$ & $10,293,300$ & $19,633,650$ & $32,011,050$ \\
\hline Fragilaria capucina & 0 & 7,500 & 450 & 600 & 150 & 5,700 & 150 & 450 \\
\hline Synedra ulna & 0 & 1,350 & 150 & 0 & 150 & 900 & 0 & 150 \\
\hline Thalassionema sp. & 21,900 & 0 & 21,450 & 0 & 450 & 0 & 0 & 0 \\
\hline $\begin{array}{c}\text { Thalassionema } \\
\text { nitzschioides }\end{array}$ & 450 & 0 & 0 & 0 & 0 & 0 & 0 & 450 \\
\hline Chaetocerous subtilis & 1,200 & 39,900 & 10,500 & 7,200 & 9,000 & 3,150 & 9,450 & 1,800 \\
\hline Leptocylindrus danicus & 9,750 & 109,350 & 17,250 & 19,800 & 43,800 & 3,000 & 26,550 & 8,700 \\
\hline L. minimus & 450 & 0 & 0 & 0 & 0 & 300 & 0 & 150 \\
\hline Stephanodiscus sp. & 0 & 48,450 & 8,700 & 5,100 & 20,400 & 300 & 8,700 & 5,250 \\
\hline $\begin{array}{l}\text { Number of species } \\
\text { identified }\end{array}$ & 49 & 72 & 56 & 54 & 47 & 38 & 43 & 49 \\
\hline Mean & 104,865 & $2,372,185$ & 963,506 & 636,897 & 337,344 & 477,126 & 470,941 & 680,623 \\
\hline Total Abundance & $5,138,400$ & $170,797,350$ & $53,956,350$ & $34,392,450$ & $15,855,150$ & $18,130,800$ & $20,250,450$ & $33,350,550$ \\
\hline $\begin{array}{l}\text { Zooplankton Abundance } \\
\qquad \text { (Bolawa, 2016) }\end{array}$ & 900,600 & $1,219,300$ & 308,250 & 222,300 & 644,700 & 70,650 & 570,250 & 303,750 \\
\hline $\begin{array}{c}\text { Ratio of Phytoplankton } \\
\text { to Zooplankton }\end{array}$ & 5.71 & 140.08 & 175.04 & 154.71 & 24.59 & 256.63 & 35.51 & 109.80 \\
\hline
\end{tabular}


Table 3. ANOVA statistics showing significant spatial variations in phytoplankton species abundance

\begin{tabular}{cccccc}
\hline & Lacustrine & Transition & Riverine & ANOVA \\
\hline Organisms & Mean \pm SD $($ Ind/L) & Mean \pm SD $($ Ind/L) & Mean \pm SD (Ind/L) & F Ratio & p \\
\hline Pediastrum sp. & $3,606.25 \pm 6,397.151$ & $2,418.75 \pm 4,673.882$ & $787.50 \pm 1,154.504$ & $3.698^{*}$ & 0.031 \\
Actinastrum hantzschii & $18.75 \pm 67.264$ & $193.75 \pm 735.485$ & $12.50 \pm 42.349$ & $3.165^{*}$ & 0.049 \\
\hline Dinophysis sp. & $2,268.75 \pm 2,668.407$ & $1,375.00 \pm 1,993.740$ & $2,643.75 \pm 3,151.029$ & $3.404^{*}$ & 0.040 \\
\hline Amphipleura jenneri & $31.25 \pm 98.701$ & 0 & 0 & $5.376^{* *}$ & 0.007 \\
\hline Pediastrum simplex & $11,187.50 \pm 13,079.383$ & $5,625.00 \pm 7,601.959$ & $3,675.00 \pm 7,153.382$ & $7.342^{* *}$ & 0.001 \\
\hline Pediastrum duplex & $2,181.25 \pm 3,739.676$ & $587.50 \pm 1,358.248$ & $343.75 \pm 957.387$ & $13.184^{* *}$ & 0.000 \\
\hline Volvox aureus & $37.50 \pm 127.048$ & $18.75 \pm 67.264$ & $162.50 \pm 356.386$ & $9.095^{* *}$ & 0.000 \\
\hline${ }^{* *}$ Highly Significant & & & & &
\end{tabular}

* Significant

Table 4. ANOVA statistics showing significant seasonal variation among phytoplankton species

\begin{tabular}{|c|c|c|c|c|}
\hline \multirow{2}{*}{ Organisms } & \multirow{2}{*}{$\begin{array}{c}\text { Dry season } \\
\text { Mean } \pm \text { SD }\end{array}$} & \multirow{2}{*}{$\begin{array}{c}\text { Wet season } \\
\text { Mean } \pm \text { SD }\end{array}$} & \multicolumn{2}{|c|}{ ANOVA } \\
\hline & & & F ratio & $\mathrm{p}$ \\
\hline Amphipleura jenneri & $250 \pm 141.42$ & 0 & $4.881^{*}$ & 0.030 \\
\hline Surirella tenara & $630 \pm 743.37$ & 0 & $4.452^{*}$ & 0.038 \\
\hline Chaetocerous subtilis & $400 \pm 187.08$ & $2,216.667 \pm 1,660.23$ & $6.485^{*}$ & 0.013 \\
\hline Leptocylindrus danicus & $886.363636 \pm 1,362.00$ & $2,865.789 \pm 4,228.21$ & $5.112^{*}$ & 0.027 \\
\hline Anabaena circinalis & $48,670 \pm 100,770.99$ & $3,255 \pm 6,032.18$ & $5.913^{*}$ & 0.018 \\
\hline Microcystis aeruginosa & $2,580 \pm 2,581.39$ & $525 \pm 375$ & $4.924^{*}$ & 0.030 \\
\hline Ceratium inflatum & $450 \pm 122.47$ & 0 & $6.146^{*}$ & 0.016 \\
\hline Euglena oxyuris & $2,335.71429 \pm 1,958.81$ & $862.5 \pm 920.85$ & $6.437^{*}$ & 0.013 \\
\hline Trichocerca caudata & 0 & $150 \pm 0$ & $4.242^{*}$ & 0.043 \\
\hline Nitzschia sp. & $350 \pm 70.71$ & $200 \pm 70.71$ & $8.889^{* *}$ & 0.004 \\
\hline Pediastrum simplex & $13,800 \pm 13,943.03$ & $4,518.75 \pm 5,455.77$ & $14.349^{* *}$ & 0.000 \\
\hline Pediastrum sp. & $5,834.21053 \pm 7,575.24$ & $1,755 \pm 1,935$ & $10.125^{* *}$ & 0.002 \\
\hline Pediastrum duplex & $3,510 \pm 4,028.95$ & $1,073.684 \pm 1,740.64$ & $8.754^{* *}$ & 0.004 \\
\hline Volvox aureus & $510 \pm 380.66$ & $150 \pm 0$ & $16.430^{* *}$ & 0.000 \\
\hline Oocystis sp. & $300 \pm 122.47$ & $777.7778 \pm 839.68$ & $7.031^{* *}$ & 0.010 \\
\hline Dinophysis sp. & $4,735.71429 \pm 3,453.75$ & $1,172.093 \pm 947.17$ & $29.942^{* *}$ & 0.000 \\
\hline
\end{tabular}

${ }^{* *}$ Highly Significant

*Significant

\section{Diversity}

The species richness recorded in the rainy season was higher than that of the dry season. Simpson's index shows a higher diversity in the dry season than wet and this agrees with the Hill's second diversity, which measured the number of very abundant species to be higher in the dry season than the wet season. Similarly, Shannon's index supports a slightly increase in the number of species and more evenness of distribution in the dry season than the wet. This is more revealed by a higher number of abundant species as Hill's first diversity index (Table 5).

Spatially, however, the maximum richness occurred in the lacustrine surface station, while the lowest occurred in the riverine surface.

The highest diversity occurred in the lacustrine bottom as revealed by both Simpson's and Shannon's indices. The station with the most evenly distributed species is the riverine bottom (Table 5).

\section{Species association}

Principal Component Analysis (PCA) based on correlation analysis, was used to reduce the component factors to those with most influence (Table 6). Twentyeight factors were found to have an Eigen value greater than 1 , that is the strongest correlation between the components and the original set of flexible quantities accounting for a cumulative variance of 87.70 but only five were selected.

Component 1 with the highest total variance of $7.56 \%$ and maximum Eigen value of 6.28 showed strongest loading (0.881) for abundance of Bacillaria paradoxa. Other species that had strong positive loadings within component 1 were Anabaena flos-aquae, Peridinium sp., Cosmarium depressum and $C$. subcrenatum. Seventeen species showed positive correlation within the first component with seven of them having high or moderate loading. Component 2 showed positive correlation for twenty species recorded, which was the highest number of positive correlations recorded (Table 6). 
294

Table 5. Spatial and temporal diversity of phytoplankton species

\begin{tabular}{ccccccccccc}
\hline & \multicolumn{2}{c}{ Temporal } & \multicolumn{2}{c}{ Spatial } \\
\cline { 3 - 10 } & & & \multicolumn{2}{c}{ Lacustrine } & \multicolumn{2}{c}{ Transition } & \multicolumn{2}{c}{ Riverine } \\
\cline { 3 - 10 } & & Dry season & Wet season & surface & bottom & surface & bottom & surface & bottom \\
\hline Richness Index & R1 & 3.11 & 3.64 & 3.26 & 3.11 & 2.71 & 2.63 & 2.49 & 2.77 \\
Simpson's Index & $\lambda$ & 0.21 & 0.61 & 0.70 & 0.41 & 0.51 & 0.45 & 0.83 & 0.91 \\
Hill's 2nd diversity & $\mathrm{N} 2$ & 4.79 & 1.64 & 1.43 & 2.46 & 1.96 & 2.20 & 1.20 & 1.10 \\
Shannon's index & H' & 1.95 & 0.89 & 0.71 & 1.14 & 1.03 & 1.02 & 0.43 & 0.27 \\
Hill's 1st diversity & $\mathrm{N} 1$ & 7.03 & 2.44 & 2.03 & 3.14 & 2.79 & 2.77 & 1.54 & 1.31 \\
Evenness Index 1 & E4 & 0.68 & 0.67 & 0.71 & 0.78 & 0.70 & 0.79 & 0.78 & 0.84 \\
Evenness Index 2 & E5 & 0.63 & 0.45 & 0.42 & 0.68 & 0.54 & 0.68 & 0.37 & 0.31 \\
\hline
\end{tabular}

Table 6. Principal component analysis for the phytoplankton species based on abundance

\begin{tabular}{|c|c|c|c|c|c|}
\hline & 1 & 2 & 3 & 4 & 5 \\
\hline Eigen value & 6.28 & 5.94 & 5.51 & 5.34 & 4.61 \\
\hline Total \% variance & 7.56 & 7.16 & 6.643 & 6.435 & 5.555 \\
\hline Cumulative variance & 7.56 & 14.72 & 21.36 & 27.80 & 33.35 \\
\hline Amphipleura jenneri & & $.310^{*}$ & & & \\
\hline Frustulia sp & $.447^{*}$ & & & & \\
\hline Naviculasp 1 & & & & $.523^{* *}$ & $.360^{*}$ \\
\hline Naviculasp 2 & & & $.711^{* *}$ & & \\
\hline Pleurosigmasp & & & & .489 & .359 \\
\hline Stauroneissp & & $.254^{*}$ & $.650^{* *}$ & & $.254^{*}$ \\
\hline Pinnularia viridis & $.398^{*}$ & & & $.290^{*}$ & \\
\hline Nitzschiasp & & $.405^{*}$ & & & \\
\hline Bacillariasp & $.447^{*}$ & & & & \\
\hline Bacillaria paradoxa & $.881^{* *}$ & & & & \\
\hline Surirella tenara & & $.323^{*}$ & $.739^{* *}$ & & $.258^{*}$ \\
\hline Surirella minuta & & & $.256^{*}$ & $.604^{* *}$ & $.424^{*}$ \\
\hline Melosira oamaruensis & & & $.544^{* *}$ & & \\
\hline Melosirasp & & & $.290^{*}$ & & \\
\hline Hyalodiscus radiatus & & & $.500^{* *}$ & & \\
\hline Rhizosoleniasp & $.736^{* *}$ & & & & $.383^{*}$ \\
\hline Thalassionemasp & & $.631^{* *}$ & & $.435^{*}$ & $.254^{*}$ \\
\hline Leptocylindrus danicus & $.436^{*}$ & & $.263^{*}$ & $.269^{*}$ & \\
\hline Stephanodiscussp & $.513^{* *}$ & & & & \\
\hline Pediastrum simplex & $.418^{*}$ & $.605^{* *}$ & & & \\
\hline Pediastrumsp & & $.472^{*}$ & & & \\
\hline Pediastrum duplex & $.308^{*}$ & $.555^{* *}$ & & $.355^{*}$ & \\
\hline Volvox globulus & $.414^{*}$ & & $.348^{*}$ & & \\
\hline Volvox aureus & & $.381^{*}$ & $.633^{* *}$ & & \\
\hline Actinastrum hantzschii & & $.347^{*}$ & $.251^{*}$ & & \\
\hline Oocystis crassa & & $.633^{* *}$ & & $.429^{*}$ & $.255^{*}$ \\
\hline Anabaena circinalis & & $.406^{*}$ & $.264^{*}$ & & \\
\hline Anabaena flos-aquae & $.777^{* * *}$ & & & & $.397^{*}$ \\
\hline Microcystissp & $.310^{*}$ & $.374^{*}$ & & & \\
\hline Artbrospirasp & $.291^{*}$ & $.598^{* *}$ & & $.359^{*}$ & $.299^{*}$ \\
\hline Trachodesmium lacustre & & $.539^{* *}$ & & $.292^{*}$ & \\
\hline Schizothrix lardacea & $.447^{*}$ & & & & \\
\hline Coelosphaerium sp & & $.386^{*}$ & & $.288^{*}$ & \\
\hline Peridinium sp & $.870^{* * *}$ & & & & \\
\hline Peridinium cinctum & & & & $.433^{*}$ & \\
\hline Peridiniopsispernardii & & & $.254^{*}$ & $.494^{*}$ & $.349^{*}$ \\
\hline Peridiniopsis thompsonii & & & $.290^{*}$ & & \\
\hline Ceratium inflatum & & $.402^{*}$ & $.702^{*}$ & & \\
\hline Dinophysissp & & $.558^{* *}$ & $.357^{*}$ & & \\
\hline Cosmarium depressum & $.777^{* * *}$ & & & & $.397^{*}$ \\
\hline Cosmarium subcrenatum & $.777^{* * *}$ & & & & $.397^{*}$ \\
\hline C. lanceolatum & & $.431^{*}$ & & $.361^{*}$ & \\
\hline Spirogyra setformis & & & $.324^{*}$ & $.546^{*}$ & $.326^{*}$ \\
\hline Euglenasp & & & $.557^{* *}$ & $.291^{*}$ & $.397^{*}$ \\
\hline Euglena acus & & & & $.556^{* *}$ & \\
\hline T. caudata & & $.385^{*}$ & & & \\
\hline Phacus pyrum & & & $.290^{*}$ & $.583^{* *}$ & \\
\hline
\end{tabular}

Note: PC loadings $<0.25$ are omitted ${ }^{*}$ Weak loading $(0.25-0.50)^{* *}$ Moderate loading $(0.50-0.75)^{* * *}$ Strong loading $(>0.75)($ Yao et al., 2014) 


\section{Discussion}

The study found that Bacillariophyta, Chlorophyta and Cyanophyta dominated the net phytoplankton of Opa Reservoir, which is in agreement with records of other studies on African tropical reservoirs, especially Nigerian reservoirs (Adeniyi, 1978; Bwalla et al., 2010; Edward and Ugwumba, 2010; Offem et al., 2011; and Atobatele, 2013). The record of the family Bacillariophyceae as most abundant is similar to the record of Abowei et al. (2012) (Koluama area) as well as Ogamba et al. (2004) (Elechi creek complex), both of Niger Delta, Nigeria; Emmanuel and Onyema, (2007) (a tropical creek in South western Nigeria); Abowei et al. (2008) (Lower Sobreiro river of the Niger Delta); Zabbey et al. (2008) (Imo river); Davies et al. (2009) (Elechi creek, Niger Delta); Achionye-Nzeh and Isimaikaye, 2010 (Ilorin reservoir) and Nkwoji et al. (2010) (Lagos lagoon, Nigeria), followed by Cyanobacteria and Chlorophyta, which were more copious in the wet season.

The maximum phytoplanktonic abundance, recorded in the lacustrine zone, might be due to stability of certain environmental variables in this zone as a result of reduced water current, restricted movement and higher transparency (Salem, 2011; Adedeji et al., 2015). The lowest abundance in spite of high species richness recorded in April 2013, a period towards the first peak of the rainy season, could possibly be an effect of the washing away of many individual phytoplankton through flooding and their dislodging from littoral vegetation hence species enrichment (Adeniyi and Adedeji, 2007). The observed irregular variation in phytoplankton distribution from surface to bottom across the three sampling stations could be as a result of high mixing and nutrient re-cycling in the reservoir column during the rainy season (Ugwumba and Ugwumba, 1993; Adedeji et al., 2015). The higher planktonic composition recorded during the rainy season may furthermore be due to an increase in ionic dilution during this period as well as an increase in nutrient inflow and introduction of organic matter (Adedeji et al., 2015).

Generally, phytoplankton has been identified to be important in bio-monitoring of trophic status as well as water quality (Townsend et al., 2000; Davies et al., 2009; Achionye-Nzeh and Isimaikaye, 2010; Offem et al., 2011). Notable bio-indicator phytoplankton species recorded were Anabaena circinalis, A. flos-aquae, Microcystis sp., Aphanocapsa litoralis and Microcystis aeruginosa, which have been reported to produce algal toxins such as microcystin, that is a hepatotoxin and can cause serious illness in both humans and some other mammals (WHO, 2009; Ugwumba et al., 2013). These species were noted to be significantly in abundance during the present study. Other pollution indicator species that were recorded in this study include oscillatoria agardhii, Phacus sp., Surirella sp., Closterium sp., Aphanocapsa sp. and Euglena sp. suggesting the likelihood of pollution in the reservoir (Ugwumba et al., 2013). Moreover, high percentage of Chlorophyceae and Cyanobacteria in a water-body, as obtained from this study, with Cyanobacteria being the second most represented taxa, is a clear indication of eutrophication (Taub, 1984; Olasehinde and Abeke, 2012). The elevated abundance of these species might have resulted from the quick increase in the supplied nutrients to the reservoir from several anthropogenic activities from the basin catchment area (Jaji et al., 2007).

\section{Conclusions}

Opa Reservoir is rich in phytoplankton, which are mostly members of Bacillariophyta (diatoms), Cyanobacteria (blue-green) and Chlorophyceae (green algae) often recorded in eutrophic lakes. As the hereby study revealed, very high abundance of the algae, recorded as compared to zooplankton abundance resulting from the increase in nutrients through continual inflow and seasonal changes, could lead to the lake deterioration with time. The lake should be therefore monitored closely.

\section{References}

Abowei JFN, Tawari CC, Hart AI, Garricks DU (2008). Fin fish species composition, abundance and distribution in the lower Sombreiro River, Niger Delta, Nigeria International Journal of Tropical Agriculture and Food Systems 2(1):46-43.

Abowei JFN, Ezekiel EN, Hansen U (2012). Effects of water pollution on phytoplankton species composition in Koluama Area, Niger Delta Area, Nigeria. International Journal of Fisheries and Aquatic Sciences 1(2):134139.

Achionye-Nzeh CG, Isimaikaiye A (2010). Fauna and flora composition and water quality of a reservoir in Ilorin, Nigeria. International Journal of Lakes and Rivers 3(1):7-15.

Adedeji AA, Komolafe OO, Akinrele OA, Adeleke O (2015). Water quality and plankton biota of Osinmo reservoir, Osun state, South-west Nigeria, Zoology and Ecology 25(2):145-153.

Adeniyi IF (1978). Studies on the physicochemical factors and planktonic algae of Lake Kainji, Nigeria. PhD Thesis. University offfe, Ile-Ife.

Adeniyi IF, Adedeji AA (2007). The Rotifera fauna of Gongola River basin, Northeast Nigeria. Ife Journal of Science 9(1):1-16.

Adesakin TA, Adedeji AA, Aduwo AI, Taiwo YF (2017). Effect of discharges from re-channeled rivers and municipal runoff on water quality of Opa reservoir, Ile-Ife, Southwest Nigeria. African Journal of Environmental Science and Technology 11(1):56-70.

Akinbuwa O, Adeniyi IF (1991). The Rotifera fauna ofOpa reservoir, Ile-Ife, Nigeria Journal of African Zoology 105(5):383-391.

Akinbuwa O, Adeniyi IF (1996). Seasonal variation, distribution and interrelationships of Rotifers in Opa Reservoir, Nigeria. African Journal ofEcology 34:351-363.

Atobatele OE (2013). Pelagic phytoplankton succession pattern in a tropical freshwater reservoir (Aiba Reservoir, Iwo, Osun state, Nigeria). Bioremediation, Biodiversity and Bioavailability 7(1):81-84.

Benedict OO, Gabriel UI (2012). Distribution and dynamics of a tropical waterfalls ecosystem. International Journal of Ecosystem 2(1):28-37.

Beyruth Z (2000). Periodic disturbances, trophic gradient and phytoplankton characteristics related to cyanobacterial growth in Guarapiranga reservoir. Sao Paulo State, Brazil. Hydrobiologia 424(1):51-60.

Bolawa OP (2016). A study of the spatial and temporal diversity and abundance of planktonic flora and fauna of Opa Reservoir, Ile Ife, 
296

Southwest, Nigeria. MSc Thesis. Department of Zoology, Obafemi Awolowo University, Ile-Ife, Osun state, Nigeria.

Bwalla RL, Ovie SI, Ajayi O, Haruna A (2010). Preliminary study on the limnology and plankton abundance in relation to fish production in some NIFFR Reservoirs. Report Opinion 2(6):7.

Codd GA (1995). Cyanobacterial toxins: Occurrence, properties and biological significance. Water Science and Technology 32:149-156.

Davies OA, Abowei JFN, Tawari CC (2009). Phytoplankton community of Elechi Creek, Niger Delta, Nigeria: A nutrient polluted tropical creek. American Journal of Applied Science 6(6):1143-1152.

EdwardJB, UgwumbaAAA (2010). Development trends and evaluation of Egbe reservoir water nutrient status in Ekiti State, Nigeria. Journal of Life Sciences $4(1): 26$.

Emmanuel BE, Onyema IC (2007). The plankton and fishes of a tropical creek in south western Nigeria. Turkish Journal of Fisheries and Aquatic Science 7:105-113.

Fawole OO, Arawomo GAO (2000). Fecundity of Sarotherodon galilaeus (Pisces: Cichlidae) in the Opa reservoir, Ile-Ife, Nigeria. Revista de Biología Tropical 48(1):201-204.

Hecky RE, Kling HJ (1981). The phytoplankton and protozooplankton of the euphotic zone of Lake Tangayinka: Species composition, biomass, chlorophyll content and spatio-temporal distribution. Limnology and Oceanography 26:548-564.

Jaji MO, Bamgbose O, Odukoya OO, Arowolo TA (2007). Water quality assessment of Ogun River, Southwest Nigeria. Environmental Monitoring and Assessment 133(1-3):473-482

Magurran AE (2004). Measuring biological diversity.2nd edition Oxford, U.K: Blackwell Science Ltd.

Margalef R (1951). Diversidad de especies en las comunidales naturales. [Diversity of species natural areas]. Public Institute of Applied Biology 9:5-27.

Menhinick EF (1964). A comparison of some species-individuals diversity indices applied to samples of field insects. Ecology 45:859-861.

Moshood KM (2009). Phytoplankton assemblage of a small, shallow, tropical African reservoir. International Journal of Tropical Biology 57(4):1009-1025.

Nkwoji JA, Onyema IC, Igbo JK (2010). Wet season spatial occurrence of phytoplankton and zooplankton in Lagos Lagoon, Nigeria. Scientific World Journal 5(5):7-14.

Nwankwo DI, Akinsoji A (1992). Epiphyte community of water hyacinth, Eichhornia crassipes (MART) Solms in coastal waters of South Western Nigeria. Archiv fur Hydrobiologie 124(4):501-511.

Offem BO, Ayotunde EO, Ikpi GU, Ada FB, Ochang SN (2011). Plankton-based assessment of the trophic state of three tropical Lakes. Journal of Environmental Protection 2:304315.

Ogamba EN, Chinda AC, Ekweozor IKE, Onwuteaka JN (2004). Water quality and phytoplankton distribution in Elechi Creek Complex of the Niger-Delta. Journal of Nigerian Environmental Society 1(2):121-130.
Olasehinde KF, Abeke AA (2012). Limnological features of Ikere Gorge Reservoir, Iseyin south-western Nigeria: Plankton composition and abundance.Journal of Biology and Environmental Sciences 2(6):20-31.

Park S, Brett MT, Oshell ET, Goldman CR (2003). Seston food quality and Daphnia production efficiencies in an oligo-mesotrophic Subalpine lake. Aquatic Ecology 37:123-136.

Pielou EC (1966). The measurement of diversity in different types of biological collections. Journal of Theoretic Biology 13:131-144.

Reynolds CS (1999). Non-determinism to probability, or N:P in the community ecology of phytoplankton: Nutrient ratios. Archiv für Hydrobiologie 146:23-35.

Salem TA (2011). Variation in phytoplankton along different zones of Aswan High Dam Reservoir. Egypt Journal of Aquatic biology and Fishery 15:87-104.

Shannon CE, Weaver W (1949). The mathematical theory of communication. Urban University, Illinois Press, Illinois.

Simciv T (2005). The role of plankton, zoobenthos, and sediment in organic matter degradation in oligotrophic and eutrophic mountain lakes. Hydrobiologia 532:69-79.

SPSS (Statistical Package for Social Sciences) (2012). Statistical Package for the Social Sciences Base 21 for Windows. Chicago, IL: SPSS

Taub DM (1984). Primate Paternalism. Burkhuys Publishers. New York, USA.

Townsend CR, Harper JD, Begon M (2000). Essentials of ecology. 3rd edition, Blackwell Science, London, UK.

Ugwumba AAA, Anago IJ, Esenowo IK (2013). The physicochemistry and plankton diversity of Awba Reservoir University of Ibadan, Ibadan Nigeria. Research Journal of Environmental and Earth Sciences 5(11):638-644.

Ugwumba AO, Ugwumba AAA (1993). A study of the physico-chemical hydrology and plankton of awba Lake in Ibadan, Nigeria. Fish Academic Communication 1:20-39.

Walsh CJ, Sharpe AK, Breen PF, Sonneman JA (2001). Effects of urbanization on streams of the Melbourne region, Victoria, Australia. I. Benthic maroinvertebrates communities. Freshwater Biology 46:535551.

WHO (2009). World Health Organization Annual Report. International Standards for drinkingwater, WHOGeneva, 1stEdition.

Yao H, Qian X, Gao H, Wang Y, Xia B (2014). Seasonal and spatial variations in heavy metals in two typical Chinese Rivers: Concentrations, environmental risks and possible sources. International Journal on Environmental Resource and Public Health 11:1186011878.

Zabbey N, Sikoki FD, Erondu J (2008). Plankton assemblages and environmental gradients in the middle reach of the Imo River, Niger Delta, Nigeria. African Journal of Aquatic Science 33(2):241-248. 